\title{
Large deviations for the right-most position of a last progeny modified branching random walk*
}

\author{
Partha Pratim Ghosh ${ }^{\dagger}$
}

\begin{abstract}
In this work, we consider a modification of the usual Branching Random Walk (BRW), where we give certain independent and identically distributed (i.i.d.) displacements to all the particles at the $n$-th generation, which may be different from the driving increment distribution. This model was first introduced by Bandyopadhyay and Ghosh [2] and they termed it as Last Progeny Modified Branching Random Walk ( $L P M-B R W)$. Under very minimal assumptions, we derive the large deviation principle $(L D P)$ for the right-most position of a particle in generation $n$. As a byproduct, we also complete the LDP for the classical model, which complements the earlier work by Gantert and Höfelsauer [7].
\end{abstract}

Keywords: branching random walk; large deviations.

MSC2020 subject classifications: 60F10; 60J80; 60G50.

Submitted to ECP on June 23, 2021, final version accepted on January 8, 2022.

Supersedes arXiv:2106.08093.

\section{Introduction}

\subsection{Background and motivation}

A branching random walk on the real line is a discrete-time stochastic process, which can be described as follows:

Let $X$ and $N$ be two random variables taking values in $\mathbb{R}$ and $\mathbb{N}$, respectively. At the 0 -th generation, we start with an initial particle at the origin. At time $n=1$, the particle dies and gives birth to a random number of offspring, distributed according to $N$. The offspring are then displaced from their parent's position by i.i.d. copies of $X$. For $n \geq 2$, the particles at generation $(n-1)$ behave independently and identically of the particles up to generation $(n-1)$.

If we denote the number of particles in generation $n$ by $N_{n}$, then from the definition, it follows that $\left\{N_{n}\right\}_{n \geq 0}$ is a Galton-Watson branching process with progeny distribution given by $N$. So the backbone of the process is a branching process tree with weighted edges. Here the weights represent the displacements of the particles relative to their respective parent. We write $|v|=n$ if an individual $v$ is in the $n$-th generation, and its position $S(v)$ is defined as the sum of the edge-weights of the unique path connecting

\footnotetext{
*The author would like to thank the Council of Scientific and Industrial Research, Government of India and the Indian Statistical Institute, Kolkata for supporting his doctoral research.

${ }^{\dagger}$ Theoretical Statistics and Mathematics Unit, Indian Statistical Institute, Delhi Centre, 7 S. J. S. Sansanwal Marg, New Delhi 110016, India. E-mail: p.pratim.10.93@gmail.com
} 
$v$ to the root. We shall call the process $\{S(v):|v|=n\}_{n \geq 0}$ a Branching Random Walk $(B R W)$.

In this article, we consider a modified version of the BRW. The modification occurs only at the last generation, where we add i.i.d. displacements of a specific form. There are two parameters of this model. One is a positively supported measure, $\mu$, and the other is a positive real number, $\theta$, which should be viewed as a scaling parameter for the extra shift we give to each particle at the $n$-th generation. The modification is as follows. At a generation $n \geq 1$, we give additional displacement to each of the particles at the generation $n$, which are of the form $\frac{1}{\theta} \log \left(Y_{v} / E_{v}\right)$, where $\left\{Y_{v}\right\}_{|v|=n}$ are i.i.d. $\mu,\left\{E_{v}\right\}_{|v|=n}$ are i.i.d. Exponential (1), and these two sequences are independent of each other and also of the BRW. This model was first introduced by Bandyopadhyay and Ghosh [2] and they refer to this new process as a Last Progeny Modified Branching Random Walk (LPM-BRW). We denote by $R_{n}$ and $R_{n}^{*} \equiv R_{n}^{*}(\theta, \mu)$ the right-most positions of the $n$-th generation particles of the BRW and the LPM-BRW, respectively, i.e.,

$$
R_{n}:=\max _{|v|=n} S(v), \quad R_{n}^{*}(\theta, \mu):=\max _{|v|=n}\left\{S(v)+\frac{1}{\theta} \log \left(Y_{v} / E_{v}\right)\right\} .
$$

The main motivation to study this model is that, due to the specific form of the additional shift at the last generation, there is a nice coupling of $R_{n}^{*}$ with a linear statistic associated with BRW, which for $\mu=\delta_{1}$ becomes the well-known Biggins' martingale (see Bandyopadhyay and Ghosh [2]). On the other hand, as $\theta$ increases, $R_{n}^{*}$ becomes closer and closer to $R_{n}$. This novel connection is in fact the reason why the model intrigued us.

Throughout this paper, we assume the followings:

(A1) The random variable $X$ is non-degenerate, i.e., $\mathbb{P}(X=t)<1$ for any $t \in \mathbb{R}$, and its moment-generating function is finite everywhere, i.e., for all $\lambda \in \mathbb{R}$,

$$
m(\lambda):=\mathbb{E}\left[e^{\lambda X}\right]<\infty .
$$

(A2) The underlying branching process is non-trivial, and the extinction probability is zero, i.e., $\mathbb{P}(N=1)<1$, and $\mathbb{P}(N=0)=0$. Also, $N$ has finite $(1+p)$-th moment for some $p>0$.

(A3) For all $k \in \mathbb{Z}$,

$$
\int_{0}^{\infty} x^{k} d \mu(x)<\infty
$$

We denote $\phi(\lambda):=\log m(\lambda)$, and $\nu(\lambda):=\phi(\lambda)+\log \mathbb{E}[N]$. Note that $\nu$ is strictly convex and infinitely differentiable under assumptions (A1) and (A2) (see Proposition A.2 of Bandyopadhyay and Ghosh [2]). We define

$$
\theta_{0}:=\inf \left\{\theta>0: \frac{\nu(\theta)}{\theta}=\nu^{\prime}(\theta)\right\} .
$$

Since $\nu(\theta)$ is strictly convex, the above set is at most singleton. If it is a singleton, then $\theta_{0}$ is the unique point in $(0, \infty)$ such that a tangent from the origin to the graph of $\nu(\theta)$ touches the graph at $\theta=\theta_{0}$. And if it is empty, then by definition $\theta_{0}$ takes value $\infty$, and there is no tangent from the origin to the graph of $\nu(\theta)$ on the right half-plane.

Under fairly general assumptions on the distribution of $X$ and $N$, Hammersley [8], Kingman [9], and Biggins [3] showed that

$$
\frac{R_{n}}{n} \rightarrow \frac{\nu\left(\theta_{0}\right)}{\theta_{0}}, \text { a.s., as } n \rightarrow \infty .
$$


Similar convergence result for LPM-BRW was proved by Bandyopadhyay and Ghosh [2]. They showed that for any $\theta>0$, almost surely

$$
\frac{R_{n}^{*}(\theta, \mu)}{n} \rightarrow c(\theta):= \begin{cases}\frac{\nu(\theta)}{\theta}, & \text { if } \theta<\theta_{0} \leq \infty \\ \frac{\nu\left(\theta_{0}\right)}{\theta_{0}}, & \text { if } \theta_{0} \leq \theta<\infty .\end{cases}
$$

Therefore, we have

$$
\begin{aligned}
& \lim _{n \rightarrow \infty} \mathbb{P}\left(\frac{R_{n}^{*}(\theta, \mu)}{n}>x\right)=0 \text { for } x>c(\theta) ; \text { and } \\
& \lim _{n \rightarrow \infty} \mathbb{P}\left(\frac{R_{n}^{*}(\theta, \mu)}{n}<x\right)=0 \text { for } x<c(\theta) .
\end{aligned}
$$

This paper investigates the exponential decay rates of these probabilities, which is in essence a large deviation (LDP) problem.

\subsection{Main results}

Let $\left\{X_{n}\right\}_{n \geq 1}$ be i.i.d. copies of $X$. We define $\mathrm{S}_{n}:=\sum_{i=1}^{n} X_{i}$. It follows from Cramér's theorem (see Dembo and Zeitouni [5]) that the laws of $\left\{S_{n} / n\right\}_{n \geq 1}$ satisfy the large deviation principle with the rate function

$$
I(x):=\sup _{\lambda \in \mathbb{R}}\{\lambda x-\phi(\lambda)\} \text { for } x \in \mathbb{R} .
$$

From Theorem 1 of Rockafellar [10], we know that $I(x)$ is strictly convex and differentiable on the interior of its effective domain $\mathcal{D}_{I}:=\{x \in \mathbb{R}: I(x)<\infty\}$ with $I^{\prime}(x)=\left(\phi^{\prime}\right)^{-1}(x)$. This implies $I^{\prime}(\mathbb{E}[X])=0$ and $\lim _{x \downarrow} \inf \mathcal{D}_{I} I^{\prime}(x)=-\infty$. Therefore, whenever $\rho:=-\log \mathbb{P}(N=1)$ is finite, there exists a unique point $a_{\theta}^{\rho} \in\left(\inf \mathcal{D}_{I}, \mathbb{E}[X]\right)$ such that a tangent from the point $(c(\theta), 0)$ to the graph of $I(x)+\rho$ touches the graph at $x=a_{\theta}^{\rho}$, i.e., $a_{\theta}^{\rho}$ satisfies

$$
\frac{I\left(a_{\theta}^{\rho}\right)+\rho}{a_{\theta}^{\rho}-c(\theta)}=I^{\prime}\left(a_{\theta}^{\rho}\right) .
$$

We denote $d(\theta):=\max \left\{c(\theta), \phi^{\prime}(\theta)\right\}$. Then we have

Theorem 1.1. The laws of $\left\{R_{n}^{*}(\theta, \mu) / n\right\}_{n \geq 1}$ satisfy the large deviation principle with the rate function

$$
\Psi_{\theta}(x):= \begin{cases}\theta x-\phi(\theta)-\log \mathbb{E}[N], & \text { if } x>d(\theta) \\ I(x)-\log \mathbb{E}[N], & \text { if } c(\theta)<x \leq d(\theta) \\ 0, & \text { if } x=c(\theta) \\ I^{\prime}\left(a_{\theta}^{\rho}\right)(x-c(\theta)), & \text { if } a_{\theta}^{\rho} \leq x<c(\theta) \text { and } \rho<\infty \\ I(x)+\rho, & \text { if } x<a_{\theta}^{\rho} \text { and } \rho<\infty \\ \infty, & \text { if } x<c(\theta) \text { and } \rho=\infty\end{cases}
$$

While proving our main result, we also observe that we can complete the LDP for $\left\{R_{n} / n\right\}_{n \geq 1}$, which was proved by Gantert and Höfelsauer [7] but only partially.

Theorem 1.2. The laws of $\left\{R_{n} / n\right\}_{n \geq 1}$ satisfy the large deviation principle with the rate function

$$
\Phi(x):= \begin{cases}I(x)-\log \mathbb{E}[N], & \text { if } x>c\left(\theta_{0}\right) \\ 0, & \text { if } x=c\left(\theta_{0}\right) \\ I^{\prime}\left(a_{\theta_{0}}^{\rho}\right)\left(x-c\left(\theta_{0}\right)\right), & \text { if } a_{\theta_{0}}^{\rho} \leq x<c\left(\theta_{0}\right) \text { and } \rho<\infty \\ I(x)+\rho, & \text { if } x<a_{\theta_{0}}^{\rho} \text { and } \rho<\infty \\ \infty, & \text { if } x<c\left(\theta_{0}\right) \text { and } \rho=\infty\end{cases}
$$


Remark 1.3. The parts (i), (ii), (iii), and (iv) of Theorem 1.2 were proved by Gantert and Höfelsauer [7], but part (v) was unsolved in their paper. As the anonymous referee pointed out, this part was recently proved by Chen and He [4]. But at the time of writing this article, the author did not know this and therefore has given an alternative proof. Also, parts (iii) and (iv) of Theorem 1.2 calculated by Gantert and Höfelsauer [7] have been simplified here. Notice here that the rate function in Theorem 1.2 is similar to that of the Branching Brownian Motion (BBM) calculated by Derrida and Shi [6]. As a result, we also see similarities in the figures in Section 2 and those in Derrida and Shi [6].

\subsection{Outline}

The article is organized as follows. In Section 2, we illustrate our main results with a few examples. We give the proofs of our main results in Section 3. Finally, in Section 4, we compare the rate function for $\left\{R_{n}^{*} / n\right\}_{n \geq 1}$ with that of $\left\{R_{n} / n\right\}_{n \geq 1}$.

\section{Examples}

As an illustration, in this section we consider two specific examples. Our first example is when $N$ takes value 2 with probability $1, X \sim \mathrm{N}(0,1), \theta=3$, and $\mu=\delta_{1}$. Then, as displayed in Figure 1, the large deviation rate function for the laws of $\left\{R_{n}^{*}\left(3, \delta_{1}\right) / n\right\}_{n \geq 1}$ is

$$
f_{1}(x)= \begin{cases}3 x-\frac{9}{2}-\log 2, & \text { if } x \geq 3 \\ \frac{x^{2}}{2}-\log 2, & \text { if } \sqrt{2 \log 2} \leq x \leq 3 \\ \infty, & \text { if } x<\sqrt{2 \log 2}\end{cases}
$$

On the other hand, if $N$ takes the value 1 with probability $1 / 2$ and 3 with probability $1 / 2$,

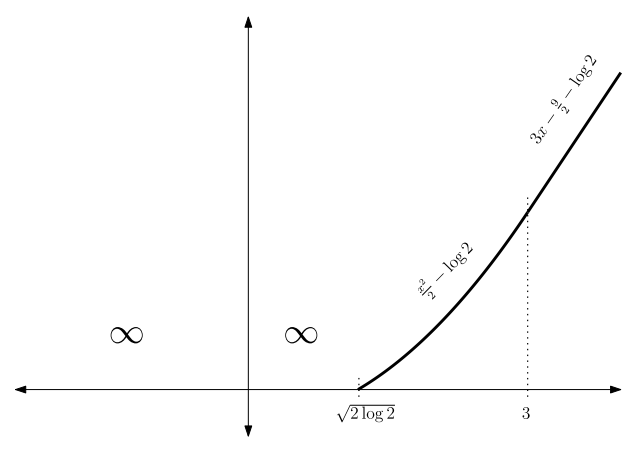

Figure 1: Graph of $f_{1}$

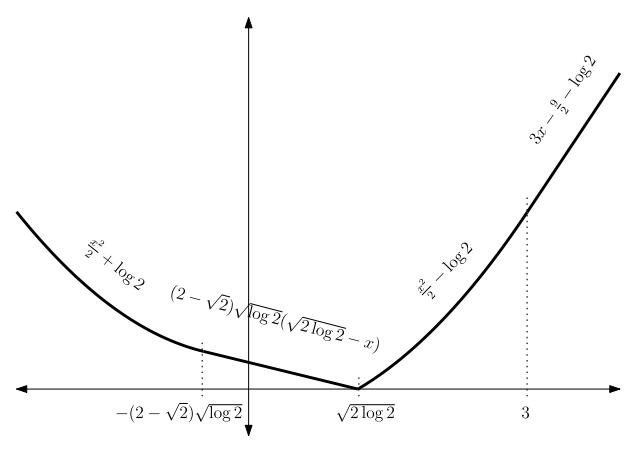

Figure 2: Graph of $f_{2}$

and $X, \theta$, and $\mu$ are as in the previous example, then, as demonstrated in Figure 2 , the large deviation rate function for the laws of $\left\{R_{n}^{*}\left(3, \delta_{1}\right) / n\right\}_{n \geq 1}$ is

$$
f_{2}(x)= \begin{cases}3 x-\frac{9}{2}-\log 2, & \text { if } x \geq 3 ; \\ \frac{x^{2}}{2}-\log 2, & \text { if } \sqrt{2 \log 2} \leq x \leq 3 \\ (2-\sqrt{2}) \sqrt{\log 2}(\sqrt{2 \log 2}-x), & \text { if }-(2-\sqrt{2}) \sqrt{\log 2} \leq x \leq \sqrt{2 \log 2} \\ \frac{x^{2}}{2}+\log 2, & \text { if } x \leq-(2-\sqrt{2}) \sqrt{\log 2}\end{cases}
$$

\section{Proofs of the main results}

\subsection{Proof of Theorem 1.1}

The general strategy to prove this theorem is to give an upper bound and a lower bound on the rate function. In most parts of the proof we will see that one bound is 
straightforward and for the other bound we decompose our LPM-BRW at an intermediate generation. In fact, the strategy for proving (iv) and (v) is similar to that used in Gantert and Höfelsauer [7]. To explain the decomposition more formally, for $v$ such that $|v|=m \leq n$, we define

$$
R_{n-m}^{*(v)}:=\max _{|u|=n, v<u}\left\{S(u)+\frac{1}{\theta} \log \left(Y_{u} / E_{u}\right)\right\}-S(v) .
$$

Here $v<u$ means $u$ is a descendant of $v$. Note that $\left\{R_{n-m}^{*(v)}\right\}_{|v|=m}$ are i.i.d. copies of $R_{n-m}^{*}$ and are independent of the BRW up to generation $m$. Now, (1.1) implies that

$$
\begin{aligned}
R_{n}^{*}=\max _{|v|=m}\left\{\max _{|u|=n, v<u}\left\{S(u)+\frac{1}{\theta} \log \left(Y_{u} / E_{u}\right)\right\}\right\} & =\max _{|v|=m}\left\{S(v)+R_{n-m}^{*(v)}\right\} \\
& \geq S\left(\tilde{v}_{m}\right)+\max _{|v|=m} R_{n-m}^{*(v)},
\end{aligned}
$$

where $\tilde{v}_{m}:=\arg \max _{|v|=m} R_{n-m}^{*(v)}$. Since $\{S(v)\}_{|v|=m}$ are identically distributed and are independent of $\left\{R_{n-m}^{*(v)}\right\}|v|=m$, we have

$$
S\left(\tilde{v}_{m}\right) \stackrel{d}{=} \mathrm{S}_{m}
$$

To prove Theorem 1.1, we also need the following lemma, which provides LDP for each of the branches of the LPM-BRW.

Lemma 3.1. Let $Y \sim \mu$ and $E \sim$ Exponential(1) be independent of each other and also independent of the random variables $\left\{X_{n}\right\}_{n \geq 1}$. Then, for any $\theta>0$, the laws of $\left\{\frac{\mathrm{S}_{n}}{n}+\frac{1}{n \theta} \log (Y / E)\right\}_{n \geq 1}$ satisfy the large deviation principle with the rate function

$$
I_{\theta}(x):= \begin{cases}I(x), & \text { if } x \leq \phi^{\prime}(\theta) \\ \theta x-\phi(\theta), & \text { if } x \geq \phi^{\prime}(\theta)\end{cases}
$$

Proof. For each $\theta>0$ and $\lambda \in \mathbb{R}$, we define

$$
\begin{aligned}
\Upsilon_{\theta}(\lambda): & =\lim _{n \rightarrow \infty} \frac{1}{n} \log \mathbb{E}\left[e^{\lambda \mathrm{S}_{n}+\frac{\lambda}{\theta} \log (Y / E)}\right] \\
& =\lim _{n \rightarrow \infty} \frac{1}{n} \log \left(e^{n \phi(\lambda)} \cdot \mathbb{E}\left[Y^{\lambda / \theta}\right] \cdot \mathbb{E}\left[E^{-\lambda / \theta}\right]\right)= \begin{cases}\phi(\lambda), & \text { if } \lambda<\theta \\
\infty, & \text { if } \lambda \geq \theta\end{cases}
\end{aligned}
$$

Its Fenchel-Legendre transform is

$$
\Upsilon_{\theta}^{*}(x):=\sup _{\lambda \in \mathbb{R}}\left\{\lambda x-\Upsilon_{\theta}(\lambda)\right\}=\sup _{\lambda<\theta}\{\lambda x-\phi(\lambda)\}=I_{\theta}(x) .
$$

Since 0 belongs to the interior of the set $\left\{\lambda \in \mathbb{R}: \Upsilon_{\theta}(\lambda)<\infty\right\}$, it follows from the GärtnerEllis theorem (see Dembo and Zeitouni [5]) that for any closed set $F$,

$$
\limsup _{n \rightarrow \infty} \frac{1}{n} \log \mathbb{P}\left(\frac{\mathrm{S}_{n}}{n}+\frac{1}{n \theta} \log (Y / E) \in F\right) \leq-\inf _{x \in F} I_{\theta}(x),
$$

and for any open set $G$,

$$
\liminf _{n \rightarrow \infty} \frac{1}{n} \log \mathbb{P}\left(\frac{\mathrm{S}_{n}}{n}+\frac{1}{n \theta} \log (Y / E) \in G\right) \geq-\inf _{x \in G, x<\phi^{\prime}(\theta)} I_{\theta}(x) .
$$

Note that since $Y$ is a positive random variable, there exists $\alpha>0$ such that $\mathbb{P}(Y>\alpha)>0$. Now, for any $x \geq \phi^{\prime}(\theta)$, we have

$$
\mathbb{P}\left(\frac{\mathrm{S}_{n}}{n}+\frac{1}{n \theta} \log (Y / E)>x\right) \geq \mathbb{P}\left(\mathrm{S}_{n}>n \phi^{\prime}(\theta)\right) \cdot \mathbb{P}(Y>\alpha) \cdot \mathbb{P}\left(E<\alpha e^{-n \theta\left(x-\phi^{\prime}(\theta)\right)}\right) .
$$


Therefore using Cramér's theorem, we get

$$
\liminf _{n \rightarrow \infty} \frac{1}{n} \log \mathbb{P}\left(\frac{\mathrm{S}_{n}}{n}+\frac{1}{n \theta} \log (Y / E)>x\right) \geq-I\left(\phi^{\prime}(\theta)\right)-\theta\left(x-\phi^{\prime}(\theta)\right)=-I_{\theta}(x) .
$$

Combining (3.5) and (3.6), we obtain that for any open set $G$,

$$
\liminf _{n \rightarrow \infty} \frac{1}{n} \log \mathbb{P}\left(\frac{\mathrm{S}_{n}}{n}+\frac{1}{n \theta} \log (Y / E) \in G\right) \geq-\inf _{x \in G} I_{\theta}(x) .
$$

This, together with (3.4), completes the proof.

Now we have all the machinery to prove Theorem 1.1.

\subsubsection{Proof of (vi)}

Proof. Take any $x<c(\theta)$ and $\epsilon \in(0, c(\theta)-x)$. Using inequality (3.2), we have

$$
\begin{aligned}
& \mathbb{P}\left(R_{n}^{*}<n x\right) \leq \mathbb{P}\left(S\left(\tilde{v}_{\lfloor\sqrt{n}\rfloor}\right)+\max _{|v|=\lfloor\sqrt{n}\rfloor} R_{n-\lfloor\sqrt{n}\rfloor}^{*(v)}<n x\right) \\
& \leq \mathbb{P}\left(\max _{|v|=\lfloor\sqrt{n}\rfloor} R_{n-\lfloor\sqrt{n}\rfloor}^{*(v)}<n(x+\epsilon)\right)+\mathbb{P}\left(S\left(\tilde{v}_{\lfloor\sqrt{n}\rfloor}\right)<-n \epsilon\right) \\
& \leq \mathbb{E}\left[\mathbb{P}\left(R_{n-\lfloor\sqrt{n}\rfloor}^{*}<n(x+\epsilon)\right)^{N_{\lfloor\sqrt{n}\rfloor}}\right]+\mathbb{P}\left(\mathrm{S}_{\lfloor\sqrt{n}\rfloor}<-n \epsilon\right) .
\end{aligned}
$$

Here $\lfloor x\rfloor$ denotes the greatest integer less than or equal to $x$, and $N_{k}$ represents the total number of particles at generation $k$. Note that $N_{k}$ is at least $2^{k}$ since $\mathbb{P}(N=1)=0$. Now since $x+\epsilon<c(\theta)$, which is the almost sure limit of $R_{n-\lfloor\sqrt{n}\rfloor}^{*} / n$, we have

$$
\begin{aligned}
& \limsup _{n \rightarrow \infty} \frac{1}{n} \log \mathbb{E}\left[\mathbb{P}\left(R_{n-\lfloor\sqrt{n}\rfloor}^{*}<n(x+\epsilon)\right)^{\left.N_{\lfloor\sqrt{n}\rfloor}\right]}\right. \\
\leq & \lim _{n \rightarrow \infty} \frac{2^{\lfloor\sqrt{n}\rfloor}}{n} \log \mathbb{P}\left(R_{n-\lfloor\sqrt{n}\rfloor}^{*}<n(x+\epsilon)\right)=-\infty .
\end{aligned}
$$

Let $\left\{t_{n}\right\}_{n \geq 1}$ be a non-negative real sequence increasing to $\infty$ such that $\phi\left(-t_{n}\right) \leq \log n$. Such a sequence exists since $\phi(\lambda)<\infty$ for all $\lambda \leq 0$. Then using Markov's inequality we obtain

$$
\begin{aligned}
\limsup _{n \rightarrow \infty} \frac{1}{n} \log \mathbb{P}\left(\mathrm{S}_{\lfloor\sqrt{n}\rfloor}<-n \epsilon\right) & \leq \lim _{n \rightarrow \infty} \frac{1}{n} \log \left(e^{-n t_{n} \epsilon} \cdot \mathbb{E}\left[e^{\left.-t_{n} \mathrm{~S}_{\lfloor\sqrt{n}\rfloor}\right]}\right)\right. \\
& =\lim _{n \rightarrow \infty}-t_{n} \epsilon+\frac{\lfloor\sqrt{n}\rfloor \phi\left(-t_{n}\right)}{n}=-\infty .
\end{aligned}
$$

Therefore, by combining (3.7), (3.8), and (3.9), we get that for $\rho=\infty$ and all $x<c(\theta)$,

$$
\lim _{n \rightarrow \infty}-\frac{1}{n} \log \mathbb{P}\left(R_{n}^{*}<n x\right)=\infty \text {. }
$$

\subsubsection{Proof of (iv) \& (v)}

Proof. (Lower bound). Take any $x<c(\theta)$ and $t \in(0,1]$. Observe that for $|v|=\lceil t n\rceil$ and $\epsilon>0$,

$$
\begin{aligned}
\mathbb{P}\left(R_{n}^{*}<n x\right) \geq & \mathbb{P}\left(S(v)+R_{\lfloor(1-t) n\rfloor}^{*(v)}<n x, N_{\lceil t n\rceil}=1\right) \\
\geq & \mathbb{P}\left(N_{\lceil t n\rceil}=1\right) \cdot \mathbb{P}\left(R_{\lfloor(1-t) n\rfloor}^{*}<n(1-t)(c(\theta)+\epsilon)\right) \\
& \cdot \mathbb{P}\left(\mathrm{S}_{\lceil t n\rceil}<n x-n(1-t)(c(\theta)+\epsilon)\right) .
\end{aligned}
$$


Here $\lceil x\rceil$ denotes the smallest integer greater than or equal to $x$. Also, note that $N_{\lceil t n\rceil}$, $S(v)$ and $R_{\lfloor(1-t) n\rfloor}^{*(v)}$ are independent of each other, which implied the last inequality. For the first term on the right-hand side, we have

$$
\lim _{n \rightarrow \infty} \frac{1}{n} \log \mathbb{P}\left(N_{\lceil t n\rceil}=1\right)=\lim _{n \rightarrow \infty} \frac{1}{n} \log \mathbb{P}(N=1)^{\lceil t n\rceil}=-\rho t .
$$

For $t=1$, the second term equals $\mathbb{P}(Y<E)>0$, and for $t \in(0,1), c(\theta)$ is the almost sure limit of $R_{\lfloor(1-t) n\rfloor}^{*} /(n(1-t))$. Therefore for all $t \in(0,1]$, we have

$$
\lim _{n \rightarrow \infty} \frac{1}{n} \log \mathbb{P}\left(R_{\lfloor(1-t) n\rfloor}^{*}<n(1-t)(c(\theta)+\epsilon)\right)=0 .
$$

Finally, for the last term, using Cramér's theorem, we get

$$
\lim _{n \rightarrow \infty} \frac{1}{n} \log \mathbb{P}\left(\mathrm{S}_{\lceil t n\rceil}<n x-n(1-t)(c(\theta)+\epsilon)\right)=-t I\left(\frac{x-(1-t)(c(\theta)+\epsilon)}{t}\right),
$$

whenever

$$
0<t \leq f(x):=\min \left\{1, \frac{c(\theta)-x}{c(\theta)-\mathbb{E}[X]}\right\} .
$$

So, by combining (3.11), (3.12), (3.13), and (3.14), and allowing $\epsilon \downarrow 0$, we obtain

$$
\liminf _{n \rightarrow \infty} \frac{1}{n} \log \mathbb{P}\left(R_{n}^{*}<n x\right) \geq-\inf _{0<t \leq f(x)}\left\{\rho t+t I\left(\frac{x-(1-t) c(\theta)}{t}\right)\right\} .
$$

Since $I((x-(1-t) c(\theta)) / t)$ is non-decreasing for $t \geq(c(\theta)-x) /(c(\theta)-\mathbb{E}[X])$, the above inequality implies

$$
\liminf _{n \rightarrow \infty} \frac{1}{n} \log \mathbb{P}\left(R_{n}^{*}<n x\right) \geq-\inf _{0<t \leq 1}\left\{\rho t+t I\left(\frac{x-(1-t) c(\theta)}{t}\right)\right\} .
$$

(Upper bound). Now, we fix any $k \in \mathbb{N}$ and define $n_{i}=\lfloor n i f(x) / k\rfloor$ for all $i=$ $0,1,2, \ldots, k$. Since $N_{n_{0}}=N_{0}=1$, for any $n \geq 2$, we have

$$
\begin{gathered}
\mathbb{P}\left(R_{n}^{*}<n x\right)=\sum_{i=0}^{k-2} \mathbb{P}\left(N_{n_{i}}<n^{2}, N_{n_{i+1}} \geq n^{2}\right) \cdot \mathbb{P}\left(R_{n}^{*}<n x \mid N_{n_{i}}<n^{2}, N_{n_{i+1}} \geq n^{2}\right) \\
+\mathbb{P}\left(N_{n_{k-1}}<n^{2}\right) \cdot \mathbb{P}\left(R_{n}^{*}<n x \mid N_{n_{k-1}}<n^{2}\right) .
\end{gathered}
$$

Using Theorem 2.5 of Gantert and Höfelsauer [7], we get that for $1 \leq i \leq k-1$,

$$
\lim _{n \rightarrow \infty} \frac{1}{n} \log \mathbb{P}\left(N_{n_{i}}<n^{2}\right)=-\frac{i f(x) \rho}{k} .
$$

On the other hand, using inequality (3.2), we have for all $\epsilon>0$ and $0 \leq i \leq k-2$,

$$
\begin{aligned}
& \mathbb{P}\left(R_{n}^{*}<n x \mid N_{n_{i}}<n^{2}, N_{n_{i+1}} \geq n^{2}\right) \\
\leq & \mathbb{P}\left(S\left(\tilde{v}_{n_{i+1}}\right)<n x-\left(n-n_{i+1}\right)(c(\theta)-\epsilon) \mid N_{n_{i}}<n^{2}, N_{n_{i+1}} \geq n^{2}\right) \\
\quad & \mathbb{P}\left(\max _{|v|=n_{i+1}} R_{n-n_{i+1}}^{*(v)}<\left(n-n_{i+1}\right)(c(\theta)-\epsilon) \mid N_{n_{i}}<n^{2}, N_{n_{i+1}} \geq n^{2}\right) \\
& \leq \mathbb{P}\left(S_{n_{i+1}}<n x-\left(n-n_{i+1}\right)(c(\theta)-\epsilon)\right)+\mathbb{P}\left(R_{n-n_{i+1}}^{*}<\left(n-n_{i+1}\right)(c(\theta)-\epsilon)\right)^{n^{2}} .
\end{aligned}
$$

Notice that $S\left(\tilde{v}_{n_{i+1}}\right)$ is independent of $N_{n_{i}}$ and $N_{n_{i+1}}$, and by (3.3), it has the same distribution as $\mathrm{S}_{n_{i+1}}$, which implied the last inequality. Now, we know that $c(\theta)$ is the almost sure limit of $R_{n-n_{i+1}}^{*} /\left(n-n_{i+1}\right)$. Therefore for any $i=0,1,2, \ldots, k-2$,

$$
\lim _{n \rightarrow \infty} \frac{1}{n} \log \mathbb{P}\left(R_{n-n_{i+1}}^{*}<\left(n-n_{i+1}\right)(c(\theta)-\epsilon)\right)^{n^{2}}=-\infty .
$$


Thus, from (3.18), we get that for any $i=0,1,2, \ldots, k-2$ and $\epsilon>0$ small enough,

$$
\begin{aligned}
& \limsup _{n \rightarrow \infty} \frac{1}{n} \log \mathbb{P}\left(R_{n}^{*}<n x \mid N_{n_{i}}<n^{2}, N_{n_{i+1}} \geq n^{2}\right) \\
\leq & \lim _{n \rightarrow \infty} \frac{1}{n} \log \mathbb{P}\left(\mathrm{S}_{n_{i+1}}<n x-\left(n-n_{i+1}\right)(c(\theta)-\epsilon)\right) \\
= & -\frac{(i+1) f(x)}{k} \cdot I\left(\frac{x-\left(1-\frac{(i+1) f(x)}{k}\right)(c(\theta)-\epsilon)}{\frac{(i+1) f(x)}{k}}\right) .
\end{aligned}
$$

For the last term of (3.16), if $f(x)=(c(\theta)-x) /(c(\theta)-\mathbb{E}[X])$, we trivially have

$$
\limsup _{n \rightarrow \infty} \frac{1}{n} \log \mathbb{P}\left(R_{n}^{*}<n x \mid N_{n_{k-1}}<n^{2}\right) \leq 0=-f(x) \cdot I(\mathbb{E}[X]) .
$$

and if $f(x)=1$, we have $x \leq \mathbb{E}[X]$. In that case, from Lemma 3.1, we have

$\limsup _{n \rightarrow \infty} \frac{1}{n} \log \mathbb{P}\left(R_{n}^{*}<n x \mid N_{n_{k-1}}<n^{2}\right) \leq \limsup _{n \rightarrow \infty} \frac{1}{n} \log \mathbb{P}\left(\mathrm{S}_{n}+\frac{1}{\theta} \log (Y / E)<n x\right)=-I(x)$.

Combining the above two inequalities, we get

$$
\limsup _{n \rightarrow \infty} \frac{1}{n} \log \mathbb{P}\left(R_{n}^{*}<n x \mid N_{n_{k-1}}<n^{2}\right) \leq-f(x) \cdot I\left(\frac{x-(1-f(x)) c(\theta)}{f(x)}\right) .
$$

Threfore, by combining (3.16), (3.17), (3.19), and (3.20), and then allowing $\epsilon \downarrow 0$ and $k \rightarrow \infty$, we obtain

$$
\limsup _{n \rightarrow \infty} \frac{1}{n} \log \mathbb{P}\left(R_{n}^{*}<n x\right) \leq-\inf _{0<t \leq 1}\left\{\rho t+t I\left(\frac{x-(1-t) c(\theta)}{t}\right)\right\} .
$$

This, together with (3.15), implies that for any $x<c(\theta)$ and $\rho<\infty$,

$$
\begin{aligned}
\lim _{n \rightarrow \infty}-\frac{1}{n} \log \mathbb{P}\left(R_{n}^{*}<n x\right) & =\inf _{0<t \leq 1}\left\{\rho t+t I\left(\frac{x-(1-t) c(\theta)}{t}\right)\right\} \\
& =\inf _{y \leq x}\left\{(\rho+I(y)) \frac{c(\theta)-x}{c(\theta)-y}\right\} \\
& =(c(\theta)-x)\left(\inf _{y \leq x}\left\{\frac{\rho+I(y)}{c(\theta)-y}\right\}\right) \\
& = \begin{cases}I^{\prime}\left(a_{\theta}^{\rho}\right)(x-c(\theta)), & \text { if } a_{\theta}^{\rho} \leq x<c(\theta) ; \\
I(x)+\rho, & \text { if } x<a_{\theta}^{\rho} .\end{cases}
\end{aligned}
$$

\subsubsection{Proof of (iii)}

Proof. This part follows from (1.3).

\subsubsection{Proof of (ii)}

Proof. Note that $c(\theta)<d(\theta)$ means $d(\theta)=\phi^{\prime}(\theta)=\nu^{\prime}(\theta)$. Therefore, $c(\theta)<d(\theta)$ occurs iff $\theta_{0}<\infty$ and $\theta>\theta_{0}$. So this part is only relevant for this range of $\theta$. 
(Upper bound). Take any $x \in(c(\theta), d(\theta)]$ and observe that

$$
\begin{aligned}
\mathbb{P}\left(R_{n}^{*}>n x\right) & =\mathbb{E}\left[\mathbb{P}\left(R_{n}^{*}>n x \mid N_{n}\right)\right] \\
& \leq \mathbb{E}\left[N_{n} \cdot \mathbb{P}\left(\mathrm{S}_{n}+\frac{1}{\theta} \log (Y / E)>n x\right)\right] \\
& =(\mathbb{E}[N])^{n} \cdot \mathbb{P}\left(\mathrm{S}_{n}+\frac{1}{\theta} \log (Y / E)>n x\right) .
\end{aligned}
$$

Since $\phi^{\prime}(\theta) \geq x>c(\theta)>\mathbb{E}[X]$, using Lemma 3.1, we get

$$
\limsup _{n \rightarrow \infty} \frac{1}{n} \log \mathbb{P}\left(R_{n}^{*}>n x\right) \leq \log \mathbb{E}[N]-I(x) .
$$

(Lower bound). For any $\alpha \in(0,1)$, using inequality (3.2), we obtain

$$
\begin{aligned}
& \mathbb{P}\left(R_{n}^{*}>n x\right) \geq \mathbb{P}\left(S\left(\tilde{v}_{\lfloor\alpha n\rfloor}\right)+\max _{|v|=\lfloor\alpha n\rfloor} R_{\lceil(1-\alpha) n\rceil}^{*(v)}>n x\right) \\
& \geq \mathbb{P}\left(S\left(\tilde{v}_{\lfloor\alpha n\rfloor}\right)>\lfloor\alpha n\rfloor x\right) \cdot \mathbb{P}\left(\max _{|v|=\lfloor\alpha n\rfloor} R_{\lceil(1-\alpha) n\rceil}^{*(v)}>\lceil(1-\alpha) n\rceil x\right) \\
& \geq \mathbb{P}\left(\mathrm{S}_{\lfloor\alpha n\rfloor}>\lfloor\alpha n\rfloor x\right) \cdot \mathbb{P}\left(N_{\lfloor\alpha n\rfloor}>\frac{1}{2} \cdot \mathbb{E}[N]^{\lfloor\alpha n\rfloor}\right) \\
& \cdot \mathbb{P}\left(\max _{|v|=\lfloor\alpha n\rfloor} R_{\lceil(1-\alpha) n\rceil}^{*(v)}>\lceil(1-\alpha) n\rceil x \mid N_{\lfloor\alpha n\rfloor}>\frac{1}{2} \cdot \mathbb{E}[N\rfloor^{\lfloor\alpha n\rfloor}\right) \\
& \geq \mathbb{P}\left(\mathrm{S}_{\lfloor\alpha n\rfloor}>\lfloor\alpha n\rfloor x\right) \cdot \mathbb{P}\left(N_{\lfloor\alpha n\rfloor}>\frac{1}{2} \cdot \mathbb{E}[N]^{\lfloor\alpha n\rfloor}\right) \\
& \cdot\left(1-\left(1-\mathbb{P}\left(R_{\lceil(1-\alpha) n\rceil}^{*}>\lceil(1-\alpha) n\rceil x\right)\right)^{\frac{1}{2} \cdot \mathbb{E}[N\rfloor^{\lfloor\alpha n\rfloor}}\right) .
\end{aligned}
$$

For any $a \in[0,1]$ and $t \geq 2$, we know that $1-(1-a)^{t} \geq a t(1-a t)$. Therefore, for all large enough $n$, we get

$$
\begin{aligned}
& \mathbb{P}\left(R_{n}^{*}>n x\right) \geq \mathbb{P}\left(\mathrm{S}_{\lfloor\alpha n\rfloor}>\lfloor\alpha n\rfloor x\right) \cdot \mathbb{P}\left(N_{\lfloor\alpha n\rfloor}>\frac{1}{2} \cdot \mathbb{E}[N]^{\lfloor\alpha n\rfloor}\right) \\
& \cdot \frac{1}{2} \cdot \mathbb{E}[N]^{\lfloor\alpha n\rfloor} \cdot \mathbb{P}\left(R_{\lceil(1-\alpha) n\rceil}^{*}>\lceil(1-\alpha) n\rceil x\right) \\
& \cdot\left(1-\frac{1}{2} \cdot \mathbb{E}[N]^{\lfloor\alpha n\rfloor} \cdot \mathbb{P}\left(R_{\lceil(1-\alpha) n\rceil}^{*}>\lceil(1-\alpha) n\rceil x\right)\right) .
\end{aligned}
$$

Note that since $c(\theta)=\nu\left(\theta_{0}\right) / \theta_{0}$, we have

$$
I(x)=\sup _{\lambda \in \mathbb{R}}\{\lambda x-\phi(\lambda)\} \geq \theta_{0} x-\phi\left(\theta_{0}\right)=\theta_{0}(x-c(\theta))+\log \mathbb{E}[N] .
$$

Now, for all $x \in(c(\theta), d(\theta)]$, we choose $\alpha_{x}$ such that

$$
0<\alpha_{x}<\frac{\theta_{0}(x-c(\theta))}{\theta_{0}(x-c(\theta))+\log \mathbb{E}[N]},
$$

which ensures $\left(1-\alpha_{x}\right) I(x)>\log \mathbb{E}[N]$. Together with (3.23), this implies

$$
\lim _{n \rightarrow \infty} \mathbb{E}[N]^{\left\lfloor\alpha_{x} n\right\rfloor} \cdot \mathbb{P}\left(R_{\left\lceil\left(1-\alpha_{x}\right) n\right\rceil}^{*}>\left\lceil\left(1-\alpha_{x}\right) n\right\rceil x\right)=0 .
$$

Therefore, for $\alpha=\alpha_{x}$, the last term on the right-hand side of (3.24) tends to 1, as $n$ tends to $\infty$. Also, assumption (A2) implies that (see Athreya and Ney [1])

$$
\lim _{n \rightarrow \infty} \mathbb{P}\left(N_{\left\lfloor\alpha_{x} n\right\rfloor}>\frac{1}{2} \cdot \mathbb{E}[N]^{\left\lfloor\alpha_{x} n\right\rfloor}\right)>0 .
$$


Thus inequality (3.24) indicates

$$
\begin{aligned}
\liminf _{n \rightarrow \infty} \frac{1}{n} \log \mathbb{P}\left(R_{n}^{*}>n x\right) \geq \lim _{n \rightarrow \infty} & \frac{1}{n} \log \mathbb{P}\left(\mathrm{S}_{\left\lfloor\alpha_{x} n\right\rfloor}>\left\lfloor\alpha_{x} n\right\rfloor x\right)+\lim _{n \rightarrow \infty} \frac{1}{n} \log \mathbb{E}[N]^{\left\lfloor\alpha_{x} n\right\rfloor} \\
& +\liminf _{n \rightarrow \infty} \frac{1}{n} \log \mathbb{P}\left(R_{\left\lceil\left(1-\alpha_{x}\right) n\right\rceil}^{*}>\left\lceil\left(1-\alpha_{x}\right) n\right\rceil x\right) .
\end{aligned}
$$

Together with Cramér's theorem, this implies

$$
\liminf _{n \rightarrow \infty} \frac{1}{n} \log \mathbb{P}\left(R_{n}^{*}>n x\right) \geq \alpha_{x}(\log \mathbb{E}[N]-I(x))+\left(1-\alpha_{x}\right) \liminf _{n \rightarrow \infty} \frac{1}{n} \log \mathbb{P}\left(R_{n}^{*}>n x\right) .
$$

Since $I(x)$ is finite for $x \in(c(\theta), d(\theta)]=\left(\phi^{\prime}\left(\theta_{0}\right), \phi^{\prime}(\theta)\right]$, using Lemma 3.1, we have

$$
\liminf _{n \rightarrow \infty} \frac{1}{n} \log \mathbb{P}\left(R_{n}^{*}>n x\right) \geq \lim _{n \rightarrow \infty} \frac{1}{n} \log \mathbb{P}\left(\mathrm{S}_{n}+\frac{1}{\theta} \log (Y / E)>n x\right)=-I(x)>-\infty .
$$

So, from (3.25), we get

$$
\liminf _{n \rightarrow \infty} \frac{1}{n} \log \mathbb{P}\left(R_{n}^{*}>n x\right) \geq \log \mathbb{E}[N]-I(x) .
$$

Combining (3.23) and (3.26), we obtain that for all $x \in(c(\theta), d(\theta)]$,

$$
\lim _{n \rightarrow \infty}-\frac{1}{n} \log \mathbb{P}\left(R_{n}^{*}>n x\right)=I(x)-\log \mathbb{E}[N] .
$$

\subsubsection{Proof of (i)}

Proof. (Upper bound). Using Markov's inequality, we obtain that for any $x \in \mathbb{R}$ and any $\lambda<\theta$,

$$
\begin{aligned}
\mathbb{P}\left(R_{n}^{*}>n x\right) \leq e^{-n \lambda x} \cdot \mathbb{E}\left[e^{\lambda R_{n}^{*}}\right] & \leq e^{-n \lambda x} \cdot \mathbb{E}\left[\sum_{|v|=n} e^{\lambda S(v)} Y_{v}^{\lambda / \theta} E_{v}^{-\lambda / \theta}\right] \\
& =e^{-n \lambda x} \cdot \mathbb{E}[N]^{n} \cdot e^{n \phi(\lambda)} \cdot \mathbb{E}\left[Y^{\lambda / \theta}\right] \cdot \Gamma\left(1-\frac{\lambda}{\theta}\right) .
\end{aligned}
$$

Since this inequality holds for all $\lambda<\theta$, we have

$$
\limsup _{n \rightarrow \infty} \frac{1}{n} \log \mathbb{P}\left(R_{n}^{*}>n x\right) \leq \lim _{\lambda \uparrow \theta}-\lambda x+\phi(\lambda)+\log \mathbb{E}[N]=-\theta x+\phi(\theta)+\log \mathbb{E}[N] .
$$

(Lower bound). For every positively supported probability $\eta$, we define

$$
A_{n}^{\eta}(\theta):=\sum_{|v|=n} e^{\theta S(v)} Z_{v},
$$

where $\left\{Z_{v}\right\}_{|v|=n}$ are i.i.d. $\eta$ and are independent of the BRW. Bandyopadhyay and Ghosh [2] showed that

$$
\theta R_{n}^{*}(\theta, \eta) \stackrel{d}{=} \log A_{n}^{\eta}(\theta)-\log E,
$$

where $E \sim$ Exponential (1) and is independent of $\left\{Z_{v}\right\}_{|v|=n}$ and also of the BRW. Therefore we get that for any $x>d(\theta)$ and any $\epsilon>0$,

$$
\begin{aligned}
\mathbb{P}\left(\frac{R_{n}^{*}(\theta, \mu)}{n}>x\right) & =\mathbb{P}\left(\frac{\log A_{n}^{\mu}(\theta)}{n \theta}-\frac{\log E}{n \theta}>x\right) \\
& \geq \mathbb{P}\left(\frac{\log A_{n}^{\mu}(\theta)}{n \theta}>d(\theta)-2 \epsilon\right) \cdot \mathbb{P}\left(-\frac{\log E}{n \theta}>x-d(\theta)+2 \epsilon\right) .
\end{aligned}
$$


Now, take any $\theta_{1} \geq \theta$ and denote $\mu_{1}$ as the distribution of $Y^{\theta_{1} / \theta}$. Then we have

$$
\left(A_{n}^{\mu}(\theta)\right)^{1 / \theta}=\left(\sum_{|v|=n} e^{\theta S(v)} Y_{v}\right)^{1 / \theta} \geq\left(\sum_{|v|=n} e^{\theta_{1} S(v)} Y_{v}^{\theta_{1} / \theta}\right)^{1 / \theta_{1}}=\left(A_{n}^{\mu_{1}}\left(\theta_{1}\right)\right)^{1 / \theta_{1}} .
$$

From (3.29), we also have

$$
\begin{aligned}
\mathbb{P}\left(\frac{R_{n}^{*}\left(\theta_{1}, \mu_{1}\right)}{n}>d(\theta)-\epsilon\right) & =\mathbb{P}\left(\frac{\log A_{n}^{\mu_{1}}\left(\theta_{1}\right)}{n \theta_{1}}-\frac{\log E}{n \theta_{1}}>d(\theta)-\epsilon\right) \\
& \leq \mathbb{P}\left(\frac{\log A_{n}^{\mu_{1}}\left(\theta_{1}\right)}{n \theta_{1}}>d(\theta)-2 \epsilon\right)+\mathbb{P}\left(-\frac{\log E}{n \theta_{1}}>\epsilon\right) .
\end{aligned}
$$

Therefore, by combining (3.30), (3.31), and (3.32), we obtain

$$
\begin{aligned}
\mathbb{P}\left(\frac{R_{n}^{*}(\theta, \mu)}{n}>x\right) \geq & \left(\mathbb{P}\left(\frac{R_{n}^{*}\left(\theta_{1}, \mu_{1}\right)}{n}>d(\theta)-\epsilon\right)-\mathbb{P}\left(-\frac{\log E}{n \theta_{1}}>\epsilon\right)\right) \\
& \cdot \mathbb{P}\left(-\frac{\log E}{n \theta}>x-d(\theta)+2 \epsilon\right) .
\end{aligned}
$$

Observe that for any $t>0$,

$$
\lim _{n \rightarrow \infty} \frac{1}{n} \log \mathbb{P}(-\log E>n t)=\lim _{n \rightarrow \infty} \frac{1}{n} \log \left(1-e^{-e^{-n t}}\right)=-t .
$$

Now, for $\theta<\theta_{0}$ or $\theta=\theta_{0}<\infty$, we take $\theta_{1}=\theta$. In that case, $d(\theta)=c(\theta)$, which implies

$$
\lim _{n \rightarrow \infty} \frac{1}{n} \log \mathbb{P}\left(\frac{R_{n}^{*}\left(\theta_{1}, \mu_{1}\right)}{n}>d(\theta)-\epsilon\right)=0 .
$$

As a result, in view of (3.33) and (3.34), we get that for $\theta<\theta_{0}$ or $\theta=\theta_{0}<\infty$,

$$
\liminf _{n \rightarrow \infty} \frac{1}{n} \log \mathbb{P}\left(\frac{R_{n}^{*}(\theta, \mu)}{n}>x\right) \geq-\theta(x-d(\theta)+2 \epsilon) .
$$

For $\theta_{0}<\theta<\infty$, we know that $c(\theta)<d(\theta)$. So choosing $\epsilon<d(\theta)-c(\theta)$, by part (ii) of the theorem, we have

$$
\lim _{n \rightarrow \infty} \frac{1}{n} \log \mathbb{P}\left(\frac{R_{n}^{*}\left(\theta_{1}, \mu_{1}\right)}{n}>d(\theta)-\epsilon\right)=-\Psi_{\theta_{1}}(d(\theta)-\epsilon)=-\Psi_{\theta}(d(\theta)-\epsilon) .
$$

Now, we choose $\theta_{1}$ large enough such that $\theta_{1} \epsilon>\Psi_{\theta}(d(\theta)-\epsilon)$, which ensures

$$
\lim _{n \rightarrow \infty} \frac{1}{n} \log \left(\mathbb{P}\left(\frac{R_{n}^{*}\left(\theta_{1}, \mu_{1}\right)}{n}>d(\theta)-\epsilon\right)-\mathbb{P}\left(-\frac{\log E}{n \theta_{1}}>\epsilon\right)\right)=-\Psi_{\theta}(d(\theta)-\epsilon) .
$$

Together with (3.33) and (3.34), this implies that for $\theta_{0}<\theta<\infty$,

$$
\liminf _{n \rightarrow \infty} \frac{1}{n} \log \mathbb{P}\left(\frac{R_{n}^{*}(\theta, \mu)}{n}>x\right) \geq-\Psi_{\theta}(d(\theta)-\epsilon)-\theta(x-d(\theta)+2 \epsilon) .
$$

Since $\epsilon>0$ can be chosen arbitrarily small and $\Psi_{\theta}$ is continuous in $[c(\theta), \infty)$, by combining (3.35) and (3.36), we get that for any $\theta>0$,

$$
\liminf _{n \rightarrow \infty} \frac{1}{n} \log \mathbb{P}\left(\frac{R_{n}^{*}(\theta, \mu)}{n}>x\right) \geq-\Psi_{\theta}(d(\theta))-\theta(x-d(\theta))=-\Psi_{\theta}(x) .
$$

Thus, by combining (3.28) and (3.37), we finally obtain that for any $x>d(\theta)$,

$$
\lim _{n \rightarrow \infty}-\frac{1}{n} \log \mathbb{P}\left(R_{n}^{*}>n x\right)=\theta x-\phi(\theta)-\log \mathbb{E}[N] .
$$




\subsection{Proof of Theorem 1.2}

For (iii) and (iv), the expression in Gantert and Höfelsauer [7] can be simplified as we did in equation (3.22). The proof of (v) is essentially the proof of the part (vi) of Theorem 1.1 verbatim. Note that the assumption $\mathbb{E}\left[N^{1+p}\right]<\infty$ in (A2) was only required for the almost sure convergence of $R_{n}^{*} / n$ and therefore is not required to prove part (v) of Theorem 1.2. But we do need $\mathbb{E}[N \log N]<\infty$ for the remaining parts, as shown in Gantert and Höfelsauer [7].

\section{Comparision with branching random walk}

We observe that for $\theta_{0} \leq \theta<\infty$, the lower large deviations for the laws of $\left\{R_{n} / n\right\}_{n \geq 1}$ and $\left\{R_{n}^{*}(\theta, \mu) / n\right\}_{n \geq 1}$ coincide. It should be noted that there is an error in deriving the lower large deviations for the laws of $\left\{R_{n} / n\right\}_{n \geq 1}$ in the work of Gantert and Höfelsauer [7]. The first term on the right-hand side of inequality 5.9 in their paper is $\exp \left(-n \rho \min \left\{1-x / x^{*}, 1\right\}+o(n)\right)$. They assumed that

$$
\rho \min \left\{1-\frac{x}{x^{*}}, 1\right\} \geq \inf _{0<t \leq 1}\left\{\rho t+t I\left(\frac{x-(1-t) x^{*}}{t}\right)\right\}
$$

which does not hold for negatively large enough $x$. The proof of (iv) and (v) of Theorem 1.1 is essentially a corrected version of their techniques.

For $\theta_{0}<\theta<\infty$, the upper large deviation for the laws of $\left\{R_{n}^{*}(\theta, \mu) / n\right\}_{n \geq 1}$ agrees with that of $\left\{R_{n} / n\right\}_{n \geq 1}$ up to $\phi^{\prime}(\theta)$.

\section{References}

[1] K. B. Athreya and P. E. Ney, Branching processes, Springer-Verlag, New York-Heidelberg, 1972, Die Grundlehren der mathematischen Wissenschaften, Band 196. MR0373040

[2] A. Bandyopadhyay and P. P. Ghosh, Right-most position of a last progeny modified branching random walk, arXiv:2106.02880 (submitted, June 2021).

[3] J. D. Biggins, The first- and last-birth problems for a multitype age-dependent branching process, Adv. in Appl. Probab. 8 (1976), no. 3, 446-459. MR0420890

[4] X. Chen and H. He, Lower deviation and moderate deviation probabilities for maximum of a branching random walk, Ann. Inst. Henri Poincaré Probab. Stat. 56 (2020), no. 4, 2507-2539. MR4164846

[5] A. Dembo and O. Zeitouni, Large deviations techniques and applications, Stochastic Modelling and Applied Probability, vol. 38, Springer-Verlag, Berlin, 2010, Corrected reprint of the second (1998) edition. MR2571413

[6] B. Derrida and Z. Shi, Large deviations for the rightmost position in a branching Brownian motion, Modern problems of stochastic analysis and statistics, Springer Proc. Math. Stat., vol. 208, Springer, Cham, 2017, pp. 303-312. MR3747671

[7] N. Gantert and T. Höfelsauer, Large deviations for the maximum of a branching random walk, Electron. Commun. Probab. 23 (2018), no. 34, 1-12. MR3812066

[8] J. M. Hammersley, Postulates for subadditive processes, Ann. Probab. 2 (1974), 652-680. MR0370721

[9] J. F. C. Kingman, The first birth problem for an age-dependent branching process, Ann. Probab. 3 (1975), no. 5, 790-801. MR0400438

[10] R. T. Rockafellar, Conjugates and Legendre transforms of convex functions, Canadian J. Math. 19 (1967), 200-205. MR0213496

Acknowledgments. This work is part of the author's Ph.D. dissertation and the author wishes to thank Antar Bandyopadhyay for suggesting the problem and also for various discussions which he had with him as the Ph.D. supervisor. The author also thanks 
Last progeny modified BRW

the anonymous referee, whose careful reading and detailed comments have helped to improve the paper. 


\section{Electronic Journal of Probability Electronic Communications in Probability}

\section{Advantages of publishing in EJP-ECP}

- Very high standards

- Free for authors, free for readers

- Quick publication (no backlog)

- Secure publication $\left(\mathrm{LOCKSS}^{1}\right)$

- Easy interface (EJMS²)

\section{Economical model of EJP-ECP}

- Non profit, sponsored by $\mathrm{IMS}^{3}, \mathrm{BS}^{4}$, ProjectEuclid ${ }^{5}$

- Purely electronic

\section{Help keep the journal free and vigorous}

- Donate to the IMS open access fund ${ }^{6}$ (click here to donate!)

- Submit your best articles to EJP-ECP

- Choose EJP-ECP over for-profit journals

\footnotetext{
${ }^{1}$ LOCKSS: Lots of Copies Keep Stuff Safe http://www. lockss.org/

${ }^{2}$ EJMS: Electronic Journal Management System http://www.vtex.lt/en/ejms.html

${ }^{3}$ IMS: Institute of Mathematical Statistics http://www.imstat.org/

${ }^{4}$ BS: Bernoulli Society http://www. bernoulli-society.org/

${ }^{5}$ Project Euclid: https://projecteuclid.org/

${ }^{6}$ IMS Open Access Fund: http://www.imstat.org/publications/open.htm
} 